\title{
The role of vitamin $C$ in pushing back the boundaries of skin aging: an ultrasonographic approach
}

This article was published in the following Dove Press journal:

Clinical, Cosmetic and Investigational Dermatology

2 September 2015

Number of times this article has been viewed

\section{Diana Crisan' \\ Iulia Roman ${ }^{2}$ \\ Maria Crisan ${ }^{2}$ \\ Karin Scharffetter- \\ Kochanek \\ Radu Badea ${ }^{3}$ \\ 'Clinic of Dermatology and Allergology, University Clinic Ulm, Ulm, Germany; ${ }^{2}$ Clinic of Dermatology and Venereology, ${ }^{3}$ Department of Clinical Imaging Ultrasound, Iuliu Hațieganu University of Medicine and Pharmacy, Cluj-Napoca, Romania}

Correspondence: Maria Crisan Clinic of Dermatology and Venereology, Iuliu Hatieganu University of Medicine and Pharmacy, Clinicilor Street No 3-5, Cluj-Napoca, Romania

Tel +40 744766496

Email mcrisan7@yahoo.com
Background: Imagistic methods stand as modern, non-invasive, and objective means of assessing the impact of topical cutaneous therapies.

Objective: This study focuses on the evaluation, by high-frequency ultrasound, of the cutaneous changes induced by topical use of a vitamin $\mathrm{C}$ complex at facial level.

Methods: A vitamin C-based solution/Placebo moisturizer cream was applied at facial level of 60 healthy female subjects according to a predetermined protocol. Ultrasonographic images (Dermascan C, $20 \mathrm{MHz}$ ) were taken from zygomatic level initially, at 40 and 60 days after therapy. The following parameters were assessed for every subject: thickness of the epidermis and dermis (mm), the number of low (LEP), medium (MEP), high echogenic pixels (HEP), and the number of LEP in the upper dermis/lower dermis (LEPs/LEPi).

Results: LEP decreased significantly in all age categories during and after therapy, but especially in the first 2 age intervals, up to the age of $50(P=0.0001)$. MEP and HEP, pixel categories that quantify protein synthesis also had an age-dependent evolution in the study, increasing significantly in all age categories but most of all in the first age interval $(P=0.002)$. Our ultrasonographic data suggest that collagen synthesis increased significantly after topical vitamin $C$ therapy, and is responsible for the increase in MEP and HEP and consequent decrease of the LEP.

Conclusion: Our study shows that topically applied vitamin $\mathrm{C}$ is highly efficient as a rejuvenation therapy, inducing significant collagen synthesis in all age groups with minimal side effects.

Keywords: ascorbic acid, rejuvenation, ultrasound, collagen synthesis

\section{Introduction}

Aging involves a complex of superimposed intrinsic and extrinsic factors that induce cumulative structural, biochemical, functional, and eventually aesthetical changes in the skin that play a major role in the social and psycho-emotional state of an individual. Our genetic material together with our life style leads to a gradual decline of skin structure and function that can be quantified at molecular, cellular, and organ level by means of modern non-invasive techniques.

The identification of the mechanisms underlying skin aging and the search for preventive or therapeutic strategies to slow down this process is a demanding and continuous challenge. This is particularly relevant as skin aging reflects general aging mechanisms of other organs and even the whole organism. ${ }^{1}$

Currently, there are several antiaging products that contain scientifically advanced ingredients with antioxidant, anti-glycation, anti-inflammatory effects that can fortify the dermal matrix and maintain the youthful skin structure and function. 
Cosmeceuticals with antioxidant properties are the most commonly indicated products designed to protect and rejuvenate the skin. Vitamin $\mathrm{C}$ is a natural antioxidant present at cutaneous level, displaying certain antioxidant, antiinflammatory, photoprotective properties, and is a known biostimulator of collagen synthesis. ${ }^{2}$

Vitamin C's instability as well as the difficulty of penetrating the cutaneous barrier that depends on the concentration and $\mathrm{pH}$ of this vitamin has been subject of several chemistry and pharmacology studies. ${ }^{3}$

The purpose of this study is to promote the use of highfrequency ultrasound as a modern, non-invasive method to reliably assess dermal changes induced by topical application of a 5\% vitamin C-based solution, complexed with Rosa moschata oil (rich in vitamins A, C, E, essential fatty acids) and proteoglycans (Benemedo, Benemedio 301, Bucharest, Romania).

\section{Materials and methods}

The current study followed three research lines including: 1) Clinical study with the establishment of experimental groups at defined age intervals and appropriate control groups. 2) Image analysis relying on ultrasonographic assessment of the changes induced following the topical application of vitamin C-derived complex in our study groups. 3) Statistical study, which by means of SPSS statistical program, assesses the variation of the ultrasonographic parameters before, during, and after therapy.

\section{Patients}

Sixty Caucasian female healthy individuals, aged between 20 and 75 years, with Fitzpatrick skin type II and III, were prospectively included in the study. The patients were enrolled from the Dermatology Clinic Cluj-Napoca, Romania and divided into three age groups: $20-35$, $35-50$, and $>50$ years.

Inclusion criteria were healthy female subjects, who requested prophylaxis or antiaging therapies. The study excluded subjects with known allergies to vitamin C, any type of facial infectious/noninfectious lesions, any type of antiaging therapies in the past 3 months, and oral contraception.

For every age interval, we included 15 subjects in the study group and 5 controls. The solution used in the study contained $5 \%$ vitamin C, proteoglycans (3\%), Rosa moschata oil $(0.3 \%)$, polysorbate $80(1 \%)$, propylene glycol ( $>70 \%$ ), water, perfume as well other components (sodium methylparaben, sodium ethylparaben, sodium propylparaben $<0.14 \%$ ). The study group was requested to topically apply $1 \mathrm{~mL}$ vitamin $\mathrm{C}$-based solution on the face every evening for 60 days and lightly massage the area until complete absorption. During this interval, they were not allowed to use any other antiaging products, apart from simple moisturizing creams. The control group was given a simple moisturizing cream, which they also had to apply at facial level daily.

Every subject was submitted to an ultrasonographic evaluation of the skin at 0,40 , and 60 days of therapy. Sonograms were acquired from the left zygomatic area. The study was approved by the Ethical Committee of the "Iuliu Hațieganu" University of Medicine and Pharmacy, Cluj-Napoca, Romania. Every subject was informed about the nature and purpose of the study and signed a written consent form before enrolling into the study. At the end of the study, probands were asked to fill in an evaluation form, regarding their satisfaction toward the use of the product.

\section{Skin ultrasound imaging}

The ultrasonographic evaluation was performed with the Dermascan device (Cortex Technologies, Hadsund, Denmark) equipped with a $20 \mathrm{MHz}$ transducer that allows sectional skin images up to a depth of $2.5 \mathrm{~cm}$. Dermascan has three major components including a transducer, an elaboration system, and a database. The ultrasonic wave is partially reflected at the boundary between adjacent structures generating echoes of different amplitudes. The intensity of the echoes is evaluated by a microprocessor and visualized as a colored twodimensional image. The color scale of echogenicity ranges from white, yellow, red, green to blue and black. On normal cutaneous sonograms, the epidermis appears as a white hyperechoic band, the dermis as a multi-colored composition (yellow, green, and/or red) and the subcutaneous tissue appears black (hypoechoic).

We applied the transducer perpendicularly on the left zygomatic area, parallel to the zygomatic arcade, after previously cleaning the cutaneous surface and applied ultrasonographic gel. The gain curve was adjusted at a value of $20 \mathrm{~dB}$ and at a speed of ultrasound at tissue level of $1,580 \mathrm{~m} / \mathrm{s}$. The obtained images were processed with an image analysis software (Dermavision, Cortex Technology). For every subject, the following parameters were measured on the obtained images before, at 40 and 60 days after therapy: thickness of the epidermis and dermis (mm), the number of low echogenic pixels (LEP), medium echogenic pixels (MEP), high echogenic pixels (HEP) as well as the 
LEPs/LEPi ratio (the ratio between the number of echogenic pixels in the upper and lower dermis). LEP quantifies the degree of cutaneous hydration, inflammatory processes, solar elastosis, and collagen degeneration. MEP and HEP quantify the structures of collagen, elastin fibers, and microfibrils. The LEPs/LEPi ratio allows an appreciation of the density and integrity of the extracellular matrix, both from the upper and lower dermis, which vary according to age, ultraviolet (UV)-ray exposure, and therapy. ${ }^{4}$

The thickness of the epidermis was obtained by calculating the mean of three measurements performed at three different sites of each image (the extremities and the center of the analyzed image). The thickness of the dermis was obtained by measuring the distance between the dermo-epidermal and the dermo-hypodermic junction at the same three different sites and by establishing the mean of the three values.

The Dermavision software is endowed with the capacity to associate the pixel amplitude with a numerical scale set ranging between 0 and 255. By selecting a certain interval from the 0-255 scale, we obtained values corresponding to a certain pixel type, present in the analyzed image. Thus, the 0-30 interval corresponds to LEP, the 50-150 interval to MEP, and the 200-255 interval to HEP. The number of LEP was determined separately in the upper (LEPs) and lower (LEPi) dermis. The boundary between these regions was the line chosen as parallel to the epidermal entrance echo and dividing the dermal image into two parts of equal thickness. The ratio of LEP number in the upper and lower dermis (LEPs/LEPi) was calculated.

\section{Statistical analysis}

The statistical analysis was performed with SPSS (v 15.0; SPSS Inc., Chicago, IL, USA). The data were analyzed by calculating the mean and standard deviation for the quantitative variables of every age group. The difference of means before and after treatment was tested using a $t$-test for paired samples. A $P$-value of $<0.05$ was considered as significant.

\section{Results}

The thickness of the epidermis increased significantly in the 20-35 age interval at 60 days of therapy compared with 40 days $(0.142 \pm 0.030$ vs $0.136 \pm 0.021, P=0.005)$ and before therapy initiation $(0.142 \pm 0.030$ vs $0.164 \pm 0.025, P=0.049)$.

As far as the dermis thickness is concerned, we did not notice any significant variation according to topical vitamin $\mathrm{C}$ complex treatment in various age groups. The number of LEP decreased significantly in all age categories during and after therapy. Of note, the most significant values were obtained in the age group ranging between 20 and 35 years $(14,319 \pm 2,424$ vs $8,447 \pm 1,811, P=0.0001)$ and the 35-50 age interval $(12,749 \pm 3,048$ vs $8,616 \pm 2,753, P=0.0001)$. In the first age interval, we notice that LEP is reduced almost by a half at 60 days of therapy $(14,319 \pm 2,424$ vs $8,447 \pm 1,811$, $P=0.0001$ ).

The number of MEP increased significantly in all age categories but most of all in the first $(3,691 \pm 1,202$ vs 5,317 \pm 746 , $P=0.002)$ and third one $(3,988 \pm 1,074$ vs $5,193 \pm 1,164$, $P=0.002$ ). The number of HEP follows the same pattern, significantly increasing in all age groups following vitamin C treatment. Of note, especially in the 20-35 age interval, the amount of pixels almost doubles at 40 days already (976 \pm 523 vs $1,629 \pm 688, P=0.006$ ) and continues to increase up to day $60(1,629 \pm 688$ vs $3,196 \pm 1,089, P<0.0001)$ as seen in Figure 1. These data suggest that collagen synthesis increased significantly, and is responsible for the increase in HEP.
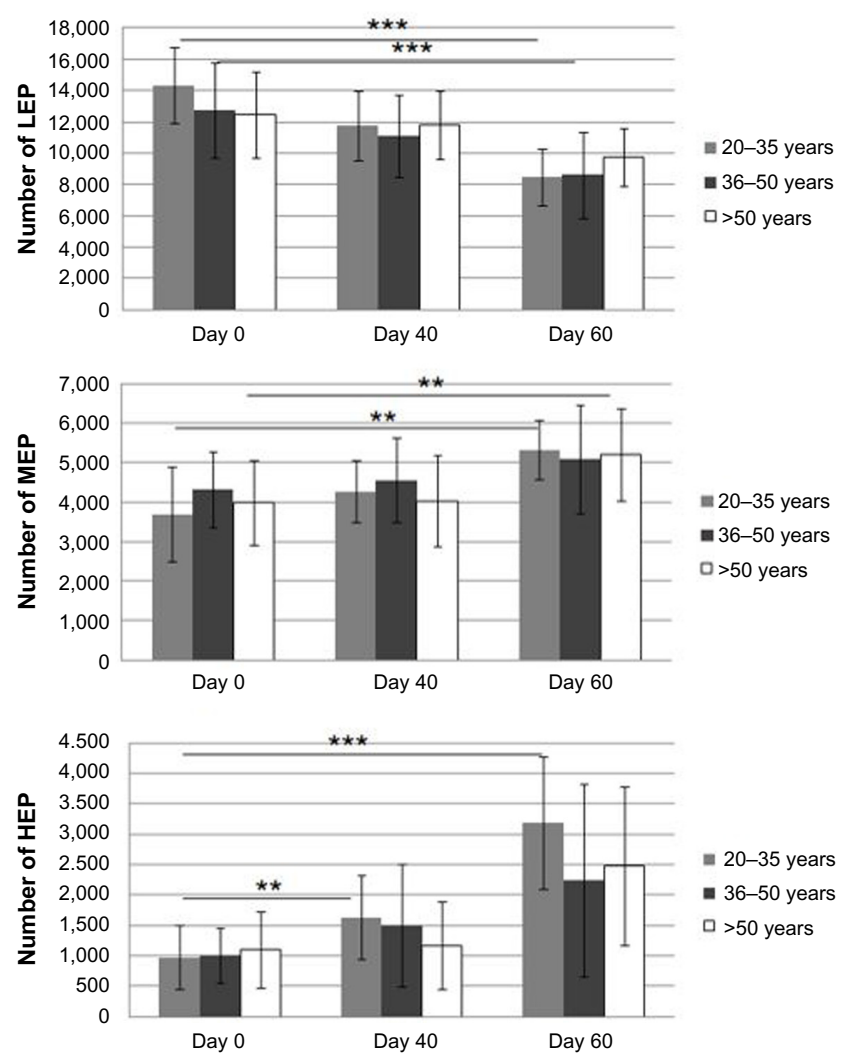

Figure I Variation of the mean number of LEP, MEP, and HEP during topically applied vitamin C-based therapy.

Notes: The figure shows a statistically significant increase in the number of MEP and HEP concomitant to the decrease in LEP. $* * P<0.0$ I, $* * * P<0.000$ I.

Abbreviations: HEP, high echogenic pixel; LEP, low echogenic pixels; MEP, middle echogenic pixel. 
The number of LEPs indicative of upper dermal changes decreases significantly after topical vitamin $\mathrm{C}$ treatment in all three age intervals; however, the decrease is most prevalent in the $20-35(4,480 \pm 1,256$ vs $2,526 \pm 707, P=0.001)$ and $>50$ age categories $(4,842 \pm 1,400$ vs $3,262 \pm 1,106$, $P<0.001)$. LEPi indicative of low pixel intensity in the lower dermis decreases significantly in the youngest age group $(9,671 \pm 1,830$ vs $6,272 \pm 1,736, P=0.04)$, and the decrease is more obvious at 40 days of therapy, followed by a more slight decrease $(P=0.04)$. The LEPs/LEPi ratio does not vary significantly, while in all age intervals it slightly increases at 40 days and thereafter decreases again.

The general variation pattern of the quantifiable ultrasonographic parameters after vitamin C-based therapy is illustrated in Figure 2.

Notably, we observed that the LEPs/LEPi ratio after topic vitamin $\mathrm{C}$ complex therapy revealed a significantly higher value in subjects belonging to phototype class III not II. We also noticed a significant increase in the LEPs/LEPi ratio in phototype II subjects in the first age interval $(0.56 \pm 0.13 \mathrm{vs}$ $0.643 \pm 0.11, P<0.05)$ and a significant decrease in the third age interval $(0.607 \pm 0.23$ vs $0.558 \pm 0.16, P<0.05)$ at 60 days post-therapy. In phototype III subjects, we noticed a significant decrease in the ratio at the end of therapy in the third $(0.727 \pm 0.33$ vs $0.524 \pm 0.25, P<0.05)$ age group which can be observed in Figure 3.

In the placebo group, we did not notice any significant variation of the mean thickness of the epidermis and a slight though not significant increase in the dermis after therapy ( $1.75 \pm 0.12 \mathrm{~mm}$ vs $1.428 \pm 0.02 \mathrm{~mm}, P=0.06$ ). The number of LEP at the upper dermal level increased in all three age groups in the dermis. This is due to an optimal hydration of the skin caused by the topical application of the moisturizing cream, which also leads to a consequent decrease in the MEP

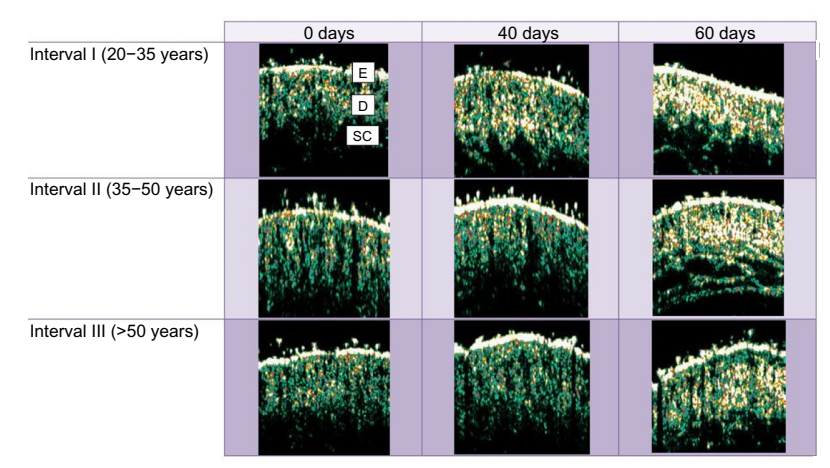

Figure 2 The variation of age-dependent ultrasonic key markers at days 0,40 , and 60 of vitamin C-based therapy. A significant increase in the hyperechoic pixels is noticed in all age intervals throughout and after topical vitamin $C$ therapy. Abbreviations: D, Dermis; E, Epidermis; SC, subcutis. and HEP at that level. The LEPs/LEPi ratio did not reveal any age- or phototype-dependent changes.

A dynamics of the variation of the ultrasonographic parameters before, during, and after therapy can be observed in Table 1.

After the first 3-4 weeks of therapy, the participants of the study groups reported several objective and subjective changes at cutaneous level such as: skin softening, visible reduction of fine wrinkles, an increase in cutaneous resilience and tonus, a good hydration as well as a bright aspect of the facial area. A discrete depigmentation of brown spots was also noticed. This is most likely due to the previously reported suppressive effects of vitamin $\mathrm{C}$ on melanin production with reduction of orthoquinones (such as dopaquinone) and subsequent prevention of the tyrosinase-dependent melanin formation. ${ }^{5}$ All abovementioned properties were maintained throughout the study. Few side effects such as local discomfort and itch were reported during the study. From all the enrolled subjects, only one patient dropped from the study due to side effects.

\section{Discussion}

A great number of topical antiaging formulations containing different natural extracts or vitamins have been claimed for their protective and rejuvenating effects. Using image analysis with high ultrasound technique, we here show that a novel vitamin $\mathrm{C}$ complex significantly improves skin structure and reconstitutes the loss of interstitial collagen, an established key marker for skin aging. This refined ultrasound technique therefore is highly suited to assess topical treatment strategies for their effectiveness to counteract the phenotype of skin aging.

Oxidative stress plays a predominant role in the induction of skin aging. ${ }^{6}$ The underlying mechanisms promoting skin aging comprise enhanced concentrations of reactive oxygen species (ROS) due to repetitive UV irradiation and reduced antioxidant defense occurring both in photoaging and in intrinsic aging. Enhanced ROS concentrations such as superoxide anion radicals as well as enhanced hydrogen peroxide concentrations drive pro-aging signaling pathways with the induction of NF-kB-dependent inflammation and AP-1-dependent matrix degradation. ${ }^{7,8}$

Both inflammation with enhanced release of ROS and pro-inflammatory cytokines like IL- $1 \alpha$ and IL-1 $\beta$ among others promote dermal matrix degradation and constitute established molecular hallmarks of cutaneous aging. ${ }^{9,10}$

The decrease in the natural cutaneous antioxidative mechanisms, overwhelmed by environmental and intrinsic 

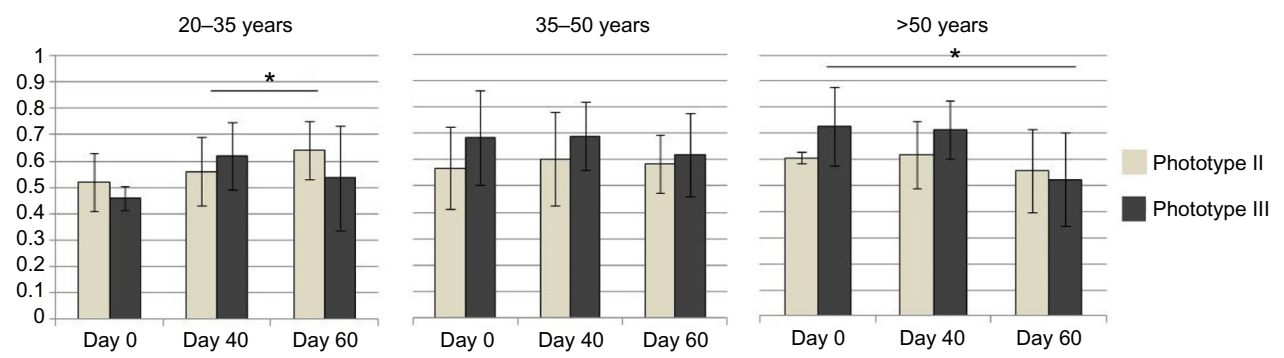

Figure 3 The variation of LEPs/LEPi ratio indicative of the dermal density according to phototype.

Notes: The lines indicate the statistically significant $(P<0.05)$ variation of the ratio during therapy. $* P<0.0$ I.

Abbreviations: LEPs, low echogenic pixels in the upper dermis; LEPi, low echogenic pixels in the lower dermis.

oxidative stress with the induction of pro-aging signaling pathways and the accumulation of structural and functional changes, plays a currently underestimated major role in intrinsic aging and photoaging. Clinical key hallmarks of ROSinduced skin aging constitute wrinkle formation, decreased resilience, skin laxity, pigmentation changes, telangiectasia, overall dehydration of the skin. Several experimental studies on animals and human have established the important role of vitamin $\mathrm{C}$ in the synthesis, remodeling, and maintenance of the dermal extracellular matrix. ${ }^{11,12}$
Vitamin $\mathrm{C}$ is a natural antioxidant that has been shown to be extremely effective for preventing and treating skin aging (it protects keratinocytes from UV radiation, stimulates the barrier function of the endothelial cells, has proven photoprotective effects). ${ }^{13,14}$

It plays an essential role in the maintenance of dermal collagen, preventing the inactivation of two important enzymes involved in the biosynthesis of collagen: hydroxylase and lysin. ${ }^{15}$ Vitamin $\mathrm{C}$ (an effective ROS scavenger) suppresses matrix-metalloproteinases responsible for

Table I The variation of the ultrasound parameters at days 0,40 , and 60 of topical vitamin C-based therapy, according to the three studied age intervals

\begin{tabular}{|c|c|c|c|c|c|c|c|}
\hline Age interval & Parameter & 0 days & $\begin{array}{l}P \text {-value } \\
0-40 \text { days }\end{array}$ & 40 days & $\begin{array}{l}P \text {-value } \\
40-60 \text { days }\end{array}$ & 60 days & $\begin{array}{l}P \text {-value } \\
0-60 \text { days }\end{array}$ \\
\hline \multirow[t]{8}{*}{$20-35$} & Epidermis & $0.142 \pm 0.030$ & 0.54 & $0.136 \pm 0.021$ & 0.005 & $0.164 \pm 0.025$ & 0.049 \\
\hline & Dermis & $|.40 \pm 0.17|$ & 0.6 & $1.383 \pm 0.143$ & 0.72 & $1.399 \pm 0.145$ & 0.91 \\
\hline & LEP & $|4,3| 9 \pm 2,424$ & 0.001 & 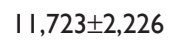 & 0.001 & $8,447 \pm I, 8 I I$ & 0.0001 \\
\hline & MEP & $3,691 \pm 1,202$ & 0.07 & $4,266 \pm 782$ & 0.003 & $5,317 \pm 746$ & 0.002 \\
\hline & HEP & $976 \pm 523$ & 0.006 & 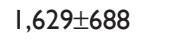 & 0.0002 & $3,196 \pm 1,089$ & $<0.0001$ \\
\hline & LEPs & $4,480 \pm I, 256$ & 0.048 & $3,628 \pm 1,010$ & 0.001 & $2,526 \pm 707$ & 0.001 \\
\hline & LEPi & $9,671 \pm 1,830$ & 0.001 & $7,430 \pm 2,113$ & 0.09 & $6,272 \pm 1,736$ & 0.04 \\
\hline & LEPs/LEPi & $0.465 \pm 0.10$ & 0.29 & $0.520 \pm 0.202$ & 0.1 & $0.423 \pm 0.14$ & 0.36 \\
\hline \multirow[t]{8}{*}{$35-50$} & Epidermis & $0.137 \pm 0.02$ & 0.2 & $0.144 \pm 0.023$ & 0.25 & $0.154 \pm 0.029$ & 0.4 \\
\hline & Dermis & $1.352 \pm 0.136$ & 0.69 & $1.340 \pm 0.106$ & 0.59 & $1.352 \pm 0.083$ & 0.98 \\
\hline & LEP & $12,749 \pm 3,048$ & 0.002 & 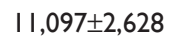 & 0.0001 & $8,616 \pm 2,753$ & 0.0001 \\
\hline & MEP & $4,319 \pm 950$ & 0.28 & $4,557 \pm|, 07|$ & 0.06 & $5,078 \pm I, 368$ & 0.044 \\
\hline & HEP & $|, 02| \pm 45 \mid$ & 0.05 & $1,510 \pm 1,008$ & 0.01 & $2,248 \pm 1,580$ & 0.01 \\
\hline & LEPs & $4,542 \pm I, 754$ & 0.5 & $4,336 \pm I, 079$ & 0.01 & $3,753 \pm I, I 76$ & 0.09 \\
\hline & LEPi & $7,575 \pm 1,498$ & 0.04 & $6,976 \pm 1,331$ & 0.2 & $6,435 \pm 1,958$ & 0.06 \\
\hline & LEPs/LEPi & $0.592 \pm 0.16$ & 0.5 & $0.619 \pm 0.07$ & 0.35 & $0.591 \pm 0.11$ & 0.98 \\
\hline \multirow[t]{8}{*}{$>50$} & Epidermis & $0.139 \pm 0.021$ & 0.3 & $0.148 \pm 0.017$ & 0.22 & $0.157 \pm 0.031$ & 0.2 \\
\hline & Dermis & $1.364 \pm 0.135$ & 0.98 & $1.365 \pm 0.17$ & 0.08 & $1.420 \pm 0.173$ & 0.25 \\
\hline & LEP & $|2,453 \pm 2,7| 3$ & 0.33 & $11,813 \pm 2,196$ & 0.001 & $9,761 \pm 1,860$ & 0.001 \\
\hline & MEP & $3,988 \pm 1,074$ & 0.9 & $4,032 \pm I,|5|$ & 0.04 & $5,193 \pm I, 164$ & 0.002 \\
\hline & HEP & $1,107 \pm 624$ & 0.75 & $\mathrm{I},|76 \pm 7| \mathrm{I}$ & 0.003 & $2,483 \pm I, 300$ & 0.001 \\
\hline & LEPs & $4,842 \pm I, 400$ & 0.29 & $4,478 \pm I, 267$ & 0.001 & $3,262 \pm 1,106$ & $<0.001$ \\
\hline & LEPi & $7,677 \pm 1,660$ & 0.15 & $6,943 \pm 1,764$ & 0.007 & $5,939 \pm 1,230$ & 0.004 \\
\hline & LEPs/LEPi & $0.64 I \pm 0.170$ & 0.88 & $0.646 \pm 0.128$ & 0.047 & $0.548 \pm 0.157$ & 0.12 \\
\hline
\end{tabular}

Note: The statistically significant variations of the parameters are marked in bold.

Abbreviations: HEP, high echogenic pixels; LEP, low echogenic pixels; MEP, middle echogenic pixel; LEPs, low echogenic pixels in the upper dermis; LEPi, low echogenic pixels in the lower dermis. 
collagen degradation, and increases the tissue inhibitor of matrix metalloproteinases, as well as messenger ribonucleic acid (mRNA) levels of collagens I and III. ${ }^{15,16}$

Boyera et al showed in their study that topically applied vitamin $\mathrm{C}$ contributes to the maintenance of the collagen quantity and density at dermal level, and is also involved in strengthening the collagen fibers. ${ }^{2}$

Other studies have shown that vitamin $\mathrm{C}$ can stimulate collagen synthesis directly by activating its transcription and stabilizing procollagen mRNA. ${ }^{17}$

In his study, Humbert et al showed a significant clinical improvement of the clinical appearance of photoexposed areas after 6 months topical application of 5\% vitamin C-based cream. ${ }^{18}$

In our study, the results have been assessed by using a global evaluation of the product's tolerability, an overall self-assessment as well as an imagistic evaluation by means of high-frequency ultrasound.

We here show that the overall assessment of the different age groups showed a great tolerability toward the product. Only $6 \%$ of the subjects complained of a discrete local discomfort, manifested by an itchy effect that lasted for more than 30 minutes. Similar reactions have earlier been described, and appear due the acidity of vitamin C. ${ }^{19}$ The vitamin $\mathrm{C}$ concentration, its $\mathrm{pH}$, and its chemical modification markedly influence the tolerability of vitamin $\mathrm{C}$, its stability, the transcutaneous transport as well as the cutaneous saturation. ${ }^{20}$ Of the esterified derivates, magnesium ascorbylphosphate is the most stable product in both solution and emulsion, followed by ascorbyl 6-phosphate and L-ascorbic acid that is the least stable product. Pinnell et al showed that daily topical application of $15 \%$ vitamin $\mathrm{C}$ at a $\mathrm{pH}$ of 3.5 or lower increases the level of ascorbic level 20 times, and cutaneous saturation is achieved in 3 days. $^{21}$

In our study, the vitamin $\mathrm{C}$ had a concentration of $5 \%$ and a $\mathrm{pH}$ of 5.5 showing a very good tolerability. The improvement of the clinical picture and sonograms (Figure 1) confirm the favorable clinical and ultrasonographic changes observed after the topical vitamin $\mathrm{C}$ treatment. Our study thus confirms recent histologic studies showing that vitamin $\mathrm{C}$ induces local structural reorganization processes. ${ }^{11,22}$

Thus, topically applied vitamin $\mathrm{C}$ induces the neosynthesis of the fibrillary structures in all age intervals. These fibrillar structures relate to de-novo synthesis of interstitial collagen as shown by trichrome stainings. These data correlate to earlier published work that collagen type I and type III synthesis is induced following topical vitamin $\mathrm{C}$ application. ${ }^{18,21}$

Of note, this remodeling with restoration of the collagen loss as well as the decrease in water binding capacity of proteoglycans in skin aging can be reliably identified by high-frequency ultrasound. The newly developed ultrasound technology could help identify several other complementary parameters of the skin (vascular structures, cutaneous elasticity), allowing a more thorough characterization of the cutaneous structure.

High-frequency ultrasound is a non-invasive technique that has been used in this study to monitor real-time kinetics on the dermal matrix. LEP, known to quantify the cutaneous hydration degree, revealed an age and therapy-dependent pattern. From the physiological point of view, the quantity of water that is uniformly bound at dermal level by proteoglycans and hyaluronic acid decreases with age. This phenomenon can be explained by the loss of the proteoglycans' hydrophilic properties, which, due to aging and glycation accumulate in the papillary dermis and form the subepidermal low echogenicity band, an ultrasonographic aging parameter quantifying local morphological changes (cutaneous laxity, elastosis). After therapy, LEP decreases significantly in all age groups, but especially in the first two age intervals, up to the age of 50 . The phenomenon is explained by the significant increase in local protein synthesis and consequent increase in the number of MEP and HEP. This specific variation of the pixels shows that collagen synthesis depends on the quantity of collagen present in the skin at the beginning of the therapy. After the age of 50, the local collagen synthesis is maintained at a reduced level, while architectural reorganization processes of the matrix are prominent.

MEP and HEP are pixel categories that quantify protein synthesis and their evolution in our study was also age dependent, being significantly amplified by vitamin $\mathrm{C}$ therapy.

MEP quantifies protein synthesis, respectively neofibrillogenesis, a process that is present and persists up to the age of 50, then decreases slightly. From histological point of view, it is considered that in physiologic conditions adult fibroblasts, to compensate the protein changes induced by aging can reactivate into fibroblasts with secretory properties.

At 40 days of therapy, we could already notice a significant increase in protein synthesis in all age categories, while at the end of treatment the increase was even more significant. The activation of collagen synthesis after the age of 50 requires prolonged topical product application, which is explained by the changes in the penetrance of the cutaneous barrier as well as by the decrease in the number of cells capable of restoring their secretory functions.

HEP quantifies mature collagen, assembled into thick fibers, that with the progression of the aging process is disposed parallelly into the profound dermis, following the 
direction of tension forces. According to our data, we notice a reorganization of the dermal matrix induced by vitamin $\mathrm{C}$ that stimulates the assemblation of the collagen fibers into the papillary dermis, at the level of Grenz zone. This aspect is also suggested by the significant decrease in LEPs in all age categories, but especially in the $>50$ age interval.

The Grenz zone which is, under physiologic conditions, a hypoechoic area that contains elastotic material becomes after vitamin $C$ therapy hyperechoic. The assembly of collagen microfibers into the papillary dermis induces a "local scar," represented by fine longitudinal bands, parallel to the cutaneous surface, which explains the increase in the cutaneous tonicity and the improvement of the fine wrinkles status. This effect is similar to the ones induced by filler substances. Kameyama et al showed that vitamin $\mathrm{C}$ induces an increase in the thickness of Grenz zone, caused by the assemblation of collagen type I microfibrils. ${ }^{23}$

Skin biopsies after topical application of vitamin $\mathrm{C}$ also showed an increase in the Grenz zone collagen as well as increased staining for mRNA for type I collagen. ${ }^{24}$

LEPi also decrease after topical vitamin $\mathrm{C}$ treatment, but the decrease is more significant in the first age interval, suggesting that in younger subjects, protein synthesis is stimulated at a deeper level as well.

The LEPs/LEPi ratio, a known imagistic parameter quantifying the dermal density, increases at 40 days posttherapy, yet not significantly, due to the significant decrease in LEPi. It then decreases at 60 days post-therapy, most significantly in the $>50$ age interval, due to the increase in MEP and HEP at the level of the papillary dermis (Grenz zone) $(P=0.047)$.

We also evaluated the LEPs/LEPi variation according to the phototype of our subjects. We noticed that the ratio had significantly higher values in phototype III subjects compared with phototype II, it increases at 40 days then decreases significantly at 60 days of therapy. Further studies are required to evaluate the different response of the phototype classes to topical antiaging therapies.

\section{Conclusion}

Our study shows that topically applied vitamin $\mathrm{C}$ (concentration of $5 \%$ and a $\mathrm{pH}$ of 5.5 in a novel complex with rosa moschata oil and proteoglycans) is highly efficient as a rejuvenation therapy, inducing significant collagen synthesis in all age groups with minimal side effects. High-frequency ultrasound can appreciate in real time and in a non-invasive manner the structural and histological changes induced by topical antiaging therapies. The quantification by using key ultrasound markers, of the tissues' remaniation processes induced by topical therapies, promotes the use of highfrequency ultrasonography for the objective assessment of the efficacy of cutaneously applied therapies.

\section{Acknowledgments}

We are grateful to Benemedo for providing the pharmaceutical products used in our study, as well as Cortex Technology Denmark for allowing us to use the Dermascan $20 \mathrm{MHz}$ device.

\section{Disclosure}

The authors report no conflicts of interest in this work.

\section{References}

1. Martin GM. Biology of aging. Aging changes in organs - tissue cells. In: Goldman L, Ausiello D, editors. Cecil Medicine. 23rd ed. Philadelphia, PA: Saunders Elsevier; 2007:chapter 22.

2. Boyera N, Galey I, Bernard BA. Effect of vitamin C and its derivatives on collagen synthesis and cross-linking by normal human fibroblasts. Int J Cosmet Sci. 1998;20(3):151-158.

3. Stamford NP. Stability, transdermal penetration, and cutaneous effects of ascorbic acid and its derivatives. $J$ Cosmet Dermatol. 2012; 11(4):310-317.

4. Man RYK, Leung SWS, Leung GPH. Effect of flavonoids on cell proliferation: comparison with estrogen. FASEB J. 2008;22:718.4.

5. Ando $\mathrm{H}, \mathrm{Kondoh} \mathrm{H}$, Ichihashi $\mathrm{M}$, et al. Approaches to identify inhibitors of melanin biosynthesis via the quality control of tyrosinase. J Invest Dermatol. 2007;127:751-761.

6. Kozina LS, Borzova IV, Arutiunov VA, et al. The role of oxidative stress in skin aging. Adv Gerontol. 2012;25(2):217-222.

7. Balistreri CR, Candore G, Accardi G, et al. NF- $\mathrm{kB}$ pathway activators as potential ageing biomarkers: targets for new therapeutic strategies. Immun Ageing. 2013;10:24.

8. Cárcamo JM, Pedraza A, Bórquez-Ojeda O, et al. Vitamin C suppresses TNF alpha-induced NF kappa B activation by inhibiting I kappa B alpha phosphorylation. Biochemistry. 2002;41(43):12995-13002.

9. Wlaschek M, Heinen G, Poswig A, et al. UVA-induced autocrine stimulation of fibroblast-derived collagenase/MMP-1 by interrelated loops of interleukin-1 and interleukin-6. Photochem Photobiol. 1994; 59(5):550-556.

10. Tilstra JS, Robinson AR, Wang J, et al. NF-kB inhibition delays DNA damage-induced senescence and aging in mice. J Clin Invest. 2012; 122(7):2601-2612.

11. Sauermann K, Jaspers S, Koop U, et al. Topically applied vitamin C increases the density of dermal papillae in aged human skin. $B M C$ Dermatol. 2004;4(1):13.

12. Fisher GJ, Kang S, Varani J, et al. Mechanisms of photoaging and chronological skin aging. Arch Dermatol. 2002;138(11):1462-1470.

13. Lin JY, Selim MA, Shea CR, et al. UV photoprotection by combination topical antioxidants vitamin C and vitamin E. J Am Acad Dermatol. 2003;48(6):866-874.

14. Barbosa NS, Kalaaji AN. CAM use in dermatology. Is there a potential role for honey, green tea, and vitamin C? Complement Ther Clin Pract. 2014;20(1):11-15

15. Nusgens BV, Humbert P, Rougier A, et al. Topically applied vitamin C enhances the mRNA level of collagens I and III, their processing enzymes and tissue inhibitor of matrix metalloproteinase 1 in the human dermis J Invest Dermatol. 2001;116(6):853-859.

16. Hantke B, Lahmann C, Venzke K, et al. Influence of flavonoids and vitamins on the MMP- and TIMP-expression of human derma fibroblasts after UVA irradiation. Photochem Photobiol Sci. 2002; 1(10):826-833. 
17. Tajima S, Pinnell SR. Ascorbic acid preferentially enhances type I and III collagen gene transcription in human skin fibroblasts. $J$ Dermatol Sci. 1996;11:250-253.

18. Humbert PG, Haftek M, Creidi P, et al. Topical ascorbic acid on photoaged skin. Clinical, topographical and ultrastructural evaluation: double-blind study vs placebo. Exp Dermatol. 2003;12(3):237-244.

19. Pumori ST. Vitamin C in dermatology. Indian Dermatol Online J. 2013; 4(2):143-146.

20. Campos PM, Gonçalves GM, Gaspar LR. In vitro antioxidant activity and in vivo efficacy of topical formulations containing vitamin $\mathrm{C}$ and its derivatives studied by non-invasive methods. Skin Res Technol. 2008; 14(3):376-380.
21. Pinnell SR, Yang H, Omar M, et al. Topical L-ascorbic acid: percutaneous absorption studies. Dermatol Surg. 2001;27(2):137-142.

22. Seité S, Bredoux C, Compan D, et al. Histological evaluation of a topically applied retinol-vitamin C combination. Skin Pharmacol Physiol. 2005;18(2):81-87.

23. Kameyama K, Sakai C, Kondoh S, et al. Inhibitory effect of magnesiumascorbyl-2-phosphate (VC-PMG) on melanogenesis in vitro and in vivo. J Am Acad Dermatol. 1996;34:29-33.

24. Fitzpatrick RE, Rostan EF. Double-blind half-face study comparing topical vitamin $\mathrm{C}$ and vehicle for rejuvenation of photodamage. Dermatol Surg. 2002;28:231-236.

\section{Publish your work in this journal}

Clinical, Cosmetic and Investigational Dermatology is an international, peer-reviewed, open access, online journal that focuses on the latest clinical and experimental research in all aspects of skin disease and cosmetic interventions. All areas of dermatology will be covered; contributions will be welcomed from all clinicians and basic science researchers globally. This journal is indexed on CAS The manuscript management system is completely online and includes a very quick and fair peer-review system, which is all easy to use. Visit http://www.dovepress.com/testimonials.php to read real quotes from published authors.

Submit your manuscript here: http://www.dovepress.com/clinical-cosmetic-and-investigational-dermatology-journal 